\title{
THE INTERNATIONAL REGULATORY REGIME FOR SATELLITE COMMUNICATIONS: THE MEANING FOR DEVELOPING COUNTRIES*
}

\author{
N. Jasentuliyana *
}

\section{INTRODUCTION}

The international regulatory regime for satellite communications has long been to work on a "first come, first served" basis which benefited the industrialized countries. Despite the general legal principle of equal access, a country wishing to put a communication satellite into the geostationary orbit had to ensure that it did not interfere with any system previously registered with the International Telecommunication Union, essentially placing a burden on the proposed new system. Since the technologically advanced countries were the first ones to set up communication satellite systems, the developing countries felt that the current registration procedures inequitably restricted their access to the geostationary orbit.

The developing countries have sought to ensure that the existing system of orbit allocation does not prevent them from implementing satellite networks. The forum in which they have sought change is the International Telecommunication Union (ITU), the specialized United Nations agency that co-ordinates orbital slots for communication satellites and registers the radio frequency assignments to avoid harmful interference between radio signals. Although the ITU does not have authority to enforce co-ordination of orbit position or radio signals, it does provide the framework for establishing the procedures for co-ordination agreed upon at specialized conferences known as World Administrative Radio

\footnotetext{
* This paper was prepared in a personal capacity and its contents should not be attributed to the United Nations.

** Director, Outer Space Affairs Division, United Nations.
}

Ko Swan Sik et al. (eds.), Asian Yearbook of International Law, Volume 2, $49-59$

(c) 1994 Kluwer Academic Publishers. Printed in the Netherlands 
Conferences (WARCs). It is at the WARCs in particular that Third World countries have sought to change regulation procedures so that they would be guaranteed access to the special orbit, the geostationary satellite orbit (GSO), in which communication satellites are placed.

\section{BENEFITS OF SATELLITE COMMUNICATIONS}

Today, it is unusual to establish long distance communications, television broadcasting, private data networks, maritime communications or disaster relief communications without satellite communications. Despite the initial financial costs, the implementation of a satellite system can be an efficient and economical solution for providing communications to widespread areas because distance has no effect on the cost of providing satellite service. Also, it is far easier to build satellite ground' stations and to use satellite circuits than to build a complete infrastructure of groundbased microwave systems, particularly over large geographic areas or difficult terrain. In addition, the implementation of a satellite system offers enormous social and economic benefits. By exploiting the broadcast capabilities, satellites can provide tele-education and tele-medicine services by transmitting educational programs and medical information directly to remote and rural villages. In terms of national sovereignty, satellites can be used to transmit vital information as well as to promote social integration by providing a means for the exchange of information between people in remote and urban areas. Also, for the 1990s, satellites are expected to be an essential element for the expansion of integrated digital networks, video programme delivery, and land and maritime mobile communications.

To implement a satellite network, however, it is necessary not only to have the financial and technical capability to launch a satellite, but also to obtain an orbit position that would adequately serve the target area and to co-ordinate the frequency assignment to avoid interference with other satellite radio signals. With the growth in the number of satellites launched, these latter requirements can present difficulties in implementing a new national satellite network because the satellites may need to be adapted to be compatible with existing systems.

\section{NATURAL LIMITATIONS}

The developing countries' concerns over accessibility are based on the natural limitations of the GSO and frequency spectrum. The GSO has a 
unique physical nature that is indispensable for relaying communication satellite signals. While in this special orbit, satellites transmit and receive radio signals for a variety of purposes including television broadcasting, long distance telephone calls, high speed data transmission, communications with remote locations and defense communications. In the GSO, satellites appear to remain stationary above the same point on the equator because they revolve in a circular orbit above the equator with the same rotational period as the Earth. Although the geostationary orbit is not the only orbit that could be used for communication satellites, it is preferred for most purposes because a satellite in the GSO has a constant view of a large area of the Earth, is constantly visible from any point within that area, and does not require that a ground antenna be continually reoriented to track the satellite. To provide coverage to a particular area, however, a satellite must be within a certain portion of the GSO. Due to the fact that most satellites serve the most highly developed areas, some portions of the GSO are very congested.

Within the GSO, satellites rely on the radio frequency spectrum for radio communications to transmit or relay information. Thus a satellite in orbit is easily affected by radio frequency interference from other satellites that use the same operating frequency. To avoid interference, limitations need to be imposed on the distance between satellites using a particular frequency. Due to the fact that the frequency spectrum can therefore only be used by a certain number of satellites to avoid problems of congestion and interference between satellite communication systems, access to the radio-frequency spectrum can present a major constraint on the use of the geostationary orbit by latecomers.

\section{EARLY DEVELOPMENTS}

In the mid-1970s, India and Indonesia were planning the establishment of their respective domestic satellite systems using fixed satellite service (FSS) radio frequencies and GSO positions. Both Intelsat and nations with existing geostationary satellites did not readily agree to make adjustments to their systems and to the internationally co-ordinated system so that India's and Indonesia's proposed systems could be accommodated. The negotiations dragged on, and India and Indonesia saw that they were in a weak and disadvantageous position as latecomers vis-à-vis the industrialized countries, which already dominated the GSO. Subsequently, the Third World camp developed a strategy to seek means to deal with these issues, which were bound to increase as more and more countries launched satellites. 
The existing regulatory regime gave priority to existing satellite systems, and the non-spacefaring nations, mostly developing countries, saw it as limiting their access to the GSO and frequency spectrum. Although the developing countries did not have any financial and technical resources to utilize satellite technologies, they wanted to ensure that they would not be precluded from access in the future by not obtaining an appropriate orbit position and frequency bandwidth now.

At the ITU, the developing countries fought for the adoption of an equitable approach to allocation. Their efforts resulted in modifications in the Radio Regulations, such as the adoption of Article 33 of the 1982 Nairobi Convention ${ }^{1}$. It laid down the principle that the geostationary orbit, a limited natural resource, must be used efficiently so that countries may have equitable access, taking into account the needs of developing countries and the geographical situation of particular countries. This was a milestone in the Third World effort to bring positive changes to the ITU regime. But despite this resolution, later entrants still faced the problem of adapting their systems to existing networks, changing satellite positions, and making adjustments in transponder frequencies.

In 1979, the ITU general WARC adopted resolutions (Resolutions 2 and 3 ) that recognized the congestion in the GSO and called on the world community to convene a conference to guarantee all nations access to the fixed satellite service radio frequencies and desired positions on the geostationary orbit. The objective of these resolutions was to fully review and revise the ITU regulations. The two-part conference known as the World Administrative Radio Conference on the Use of the Geostationary Orbit and the Planning of the Space Services Utilizing It, WARC-ORB (also known as Space WARC) of 1985 and 1988 set out to realize this objective.

\section{WARC-ORB 85}

The commencement of the 1985 WARC-ORB was characterized by a fair amount of political dissension and polarization between the developing countries and the industrialized countries. Developing countries argued for a priori planning with fixed orbital positions for most of the Fixed Satellite Service (FSS) bands to guarantee GSO access. The developed countries believed that the existing regulatory regime was adequate and that access to the GSO would continue to be available through technological advances. ${ }^{2}$ It may have been true that advancing technology

1. International Telecommunication Convention, Final Protocol, Additional Protocols, Optional additional Protocols, Resolution and Opinions (Nairobi, 1982). 
had the ability to relieve congestion on the GSO, but the developing countries saw that technology was controlled by the developed countries, and therefore, the developing countries preferred political solutions over technical ones.

Despite political tension, the 1985 WARC agreed on the general principle of an allotment plan limited to the expansion bands for FSS. The planning approach for these FSS bands would permit each country to satisfy requirements for national service from at least one orbital position within a predetermined arc and with a total of $800 \mathrm{MHz}$ of bandwidth in a defined service area and within predetermined bands (specifically in the 6/4, 14/ 11-12 and 20/30 GHz bands). This arc allotment plan, or predetermined arc (PDA), allocated arc segments rather than specific orbital locations, and tried to guarantee access to all countries. The 1985 Conference also proposed that multilateral planning meetings (MPMs) would provide a mechanism for concerned nations to solve regional issues as equals by facilitating the co-ordination process between a country with a proposed new satellite system and others already using the frequency bands and orbital arcs concerned. Yet, while countries with existing assignments would be expected to make minor adjustments, if necessary, the essential burden was still on the proposed new satellite system.

The outline agreed at WARC-ORB 85 did not constitute international law, but was a report to the second session which then had to discuss details on implementation.

\section{LEGAL PRINCIPLES}

The 1967 Outer Space Treaty provides that outer space is "free for use by all States" and "is not subject to national appropriation by claim of sovereignty, by means of use or occupation, or by any other means." Adopting the view of most States that the geostationary orbit is a part of outer space, it follows that the use of the GSO should be free for use by all States.

However, the effective use of the GSO for satellite communications requires satellite communication systems to be protected from interference from other satellites and radio signals/frequencies. Therefore, the freedom of use of the orbit needs to be regulated to prevent such interference. The ITU is vested with the responsibility for technical co-ordination of communication satellites to prevent such interference. The Interna-

2. On the positions of the developing and developed countries at Space WARC 1985, see M.L. SMITH, "A new era for the international regulation of satellite communications", 14 Annals of Air and Space Law (1989) pp. 449, 450. 
tional Telecommunication Convention and the Radio Regulations, which are negotiated and revised regularly in the ITU, constitute binding treaties of international law. They contain policies and procedures for co-ordinating international telecommunications, including satellite communications. Resolutions and recommendations which are not legally binding are also attached to the Radio Regulations.

The contention lies in the theoretical conflict between the general principle of space law as established in the Outer Space Treaty, providing access and use of outer space by all States, and the co-ordination procedures established in the Radio Regulations necessitated to ensure interference-free operations. The developing countries see the Radio Regulations as a regime favouring existing satellite systems at the expense of future satellite systems. Article 13 of the Radio Regulations, for example, provides that a geostationary satellite frequency and position assignment, once registered with ITU's International Frequency Registration Board, will be protected against interference from new systems indefinitely as long as it is continuously used. Resolution 4 adopted at the 1979 WARC attempted to reconcile these two legal provisions, but it also demonstrated that imposing limitations on the period of use was difficult.

Resolution 4 provided that States which registered geostationary positions and frequency assignments were to specily the "period of operation" of the assignment which was to be "limited to that for which the satellite network was designed." The period of validity was limited to the planned lifetime of the satellite network, and not necessarily to the lifetime of any satellite; the resolution in fact specifically noted that a satellite could be replaced by another identical satellite without any effect on the assignment. Moreover, countries were able to extend the period of validity provided that new satellites did not increase the probability of interference with other registered assignments. Other considerations stipulated in the Resolution effectively kept the system of indefinite geostationary assignments intact.

Resolution 4 was not intended to change international space law; it was more a voluntary mechanism for encouraging countries to release assignments that were not needed. Satellite communication networks, though, are rarely set up for a limited duration, and generally are intended for permanent use, with the organizations and hardware evolving to meet the evolving functions of the system. Countries with networks have no interest in periodically shuffling their satellites around and reorienting all of their Earth stations merely to avoid permanent occupancy. The issue of indefinite period of assignments was to be dealt with at WARC-ORB 88. 
The legal debate, though, goes beyond the question of access to the GSO. ${ }^{3}$ The difference in approaches to the development of law runs at the core of this debate. The civil law tradition of continental Europe, generally followed by the developing countries, establishes general principles and then applies them to specific problems, whereas the Anglo-American common law tradition develops rules of law from precedent, and develops general principles gradually through resolution of specific practical problems as they arise. The ITU procedures basically follow the common law approach, having an ad hoc technical problem-solving mechanism. But the technical problem-solving mechanism tends to be dominated by experts from technologically advanced countries. The developing countries are instead pushing for immediate elaboration and application of general principles based on the equality of States.

More recently, a debate over the legal criteria on the use of the GSO has emerged. Various ITU resolutions include references to both "equal use" and "equitable use" of the GSO, ${ }^{4}$ using these terms interchangeably, while in strict, legal terms they represent different concepts. For example, the 1979 WARC Resolution 3 that was titled "Equitable Uses" called for the "equal right" of all States to use the orbit and spectrum resource. Also, Resolution 3 identified the goal of the 1985/1988 WARC as being to "guarantee in practice for all countries equitable access. ${ }^{n}$ In addition, the 1982 ITU Convention referred to the need of countries to have "equitable access" to the orbit.

The term "equitable" does not necessarily connote equal, which means the same or uniform treatment. The request for "equitable" treatment is generally understood by the ITU as a claim for equity or fairness of treatment taking into consideration facts and special circumstances of each case, and not unqualified equal treatment in all situations. ${ }^{6}$ According to the Outer Space Treaty, ${ }^{7}$ however, outer space is free for use by all countries irrespective of economic or scientific development ${ }^{8}$ without discrimination of any kind. In the 1982 ITU Convention, ${ }^{9}$ Articles 10 and

3. N. JASENTULIYANA and R. CHIPMAN, "The current legal regime of the geostationary orbit and prospects for the future", 17:6 Acta Astronautica, pp. 600-601.

4. C. CHRISTOL, "The legal status of the geostationary orbit in light of the 1985-1988 activities of the ITU", Proceedings of the Thirty-Second Colloquium on the Law of Outer Space, 11-15 October 1989, pp. 215, 216.

5. Ibid.

6. See loc. cit. n.4, at n. 6 (analyzing the concept of equity and implications for an allotment plan).

7. Treaty on Principles Governing the Activities of States in the Exploration and Use of Outer Space, Including the Moon and Other Celestial Bodies, 10 October 1967, 610 UNTS 105.

8. Id. at Article 1.

9. Supra n.1. 
33 require that some special treatment be given to the developing countries that did not currently possess the scientific, technological and economic means to utilize the geostationary orbit. Accordingly, the international law of the Radio Regulations gives special consideration to promote fair and equitable treatment for access to the GSO. Some international law specialists concerned with space law would like to see more clarification on the use of terms so that complications may be prevented.

\section{WARC-ORB 88}

The 1988 WARC, compared to the 1985 session, commenced with less political tension and ended successfully with a new addition to the international regulatory regime for radio communications that provided for an allotment plan for predetermined arcs and bands. The countries reviewed and revised existing resolutions and recommendations associated with international radio regulations and adopted new Resolutions and Recommendations in the Final Act. ${ }^{10}$ These Acts, which entered into force on 16 March 1990, marked an important step for the Third World in regulating the use of the frequency spectrum and the geostationary orbit, particularly for FSS. Two regulatory regimes emerged for FSS: an allotment plan, and a regime for unplanned bands.

The primary result of WARC-ORB 88 is the establishment of a flexible predetermined arc allotment plan for FSS that permits at least one national orbital allotment and $800 \mathrm{MHz}$ of frequency bandwidth. It is designed to assist ITU members in obtaining a new allotment, in converting an allotment into an assignment, in accommodating subregional systems, and in resolving incompatibilities between existing systems. Although the allotment plan gives each country one nominal orbital position, some countries, including Brazil, Canada, USSR and the United States, received two nominal orbital locations because these countries have service areas too large for one orbital location.

In addressing the issue of indefinite period of occupancy, the plan called for existing ${ }^{11}$ systems (that had initiated the ITU co-ordinating process prior to WARC-ORB 88) to follow provisions of the allotment plan which imposed a time limit for use: a non-renewable period with a dead-

10. Final Act, adopted by the Second Session of the World Administrative Radio Conference on the Use of the Geostationary Satellite Orbit and the Planning of the Space Services Utilizing It (ORB-88), Geneva 1988.

11. S. DOYLE, "Space law and the geostationary orbit: The ITU's WARC-ORB concluded", $17 \mathrm{~J}$. Space L. (1989) p. 13. 
line of 15 March $2010 .{ }^{12}$ But it did not specify how existing systems were to be integrated in the plan.

Also, some countries were concerned that the allocated spectrum might not actually be used by the planned allotments, so WARC-ORB 88 established provisions for use of the orbit outside the plan, called additional use provisions, so that unused planned allotments could be utilized. To protect national allotments, however, additional uses shall have a maximum period of validity of 15 years and shall not be incompatible with any allotment or assignment in the allotment plan. This restriction was included to relieve the developing countries' concerns that the industrialized countries' additional uses would preclude the implementation of their allotments.

As for the regulatory regime of unplanned bands, the basic attributes of the former regulatory regime for FSS were preserved. The method of gaining access to the unplanned bands follows the procedures of Articles 11 and 13 of the Radio Regulations. There were a few changes made, though, to mitigate some of the perceived unfaimess of the first-comefirst-served approach. The simplification of procedures in the regime, such as those for network co-ordination and notification, for example, is a significant improvement. ${ }^{13}$

One of the changes in provisions from the 1985 WARC was the diminished role of the MPMs. The 1985 session envisioned MPMs as a regular method for gaining access to many of the FSS bands. The 1988 session, however, altered the requirements for MPMs, so that they would take place only in exceptional cases when major difficulties took place in obtaining co-ordination in specific FSS bands.

The Conference also adopted a feeder link plan for Broadcasting Satellite Systems (BSS), thereby allowing all countries to have a BSS feeder allotment. The allocation of frequencies for Satellite Sound Broadcasting and High Definition Television remained open for future consideration.

The allotment plan ensured that developing countries will not be entirely precluded from the GSO, and WARC-ORB 88 adopted this plan while introducing only minimal changes to the international regime for the co-ordination of satellites for the older FSS bands. The outcome, particularly for FSS, represented a reasonable compromise between maximum efficiency in use of the GSO and guaranteeing the developing countries' access to limited resource.

12. B.J. TAYLOR, Depoliticizing Space WARC Satellite Communications (January 1989) pp. 2829.

13. M.L. SMITH, International Regulation of Satellite Communication (Utrecht Studies in Air and Space Law, Volume 7 (1990)), p. 222. 


\section{INTERESTS OF DEVELOPING COUNTRIES}

Despite the allotment plan, however, WARC-ORB 88 was perceived as not fully meeting the Third World's expectations. The allotment plan is limited to only one service (the FSS) ${ }^{14}$ out of seventeen space services. The allotment plan defines only a nominal position in a predetermined arc, with the planned portion covering less than one percent of the total spectrum allocated to space services. The "first come, first served" basis in the occupation of the geostationary orbit is still more or less intact. ${ }^{15}$ WARC-ORB 88 also reduced the use of the MPM process formulated in 1985. For some Third World countries, the restraints on the use of the MPM may limit their ability to resolve regional co-ordination and technical problems.

In addition, the WARC-ORB 88 did not resolve the equatorial States' claim to sovereignty or preferential rights to the GSO as presented in the 1976 Bogota Declaration. Several equatorial countries claimed that due to their special geographical relationship to the GSO they were entitled to exercise sovereignty over corresponding parts of the GSO. Other countries, however, expressed concern that any preferential access would preclude equitable access. It was also argued by other countries, including the developed countries, that any claim of sovereignty over the GSO would contradict the principles of the Outer Space Treaty, particularly Article II, which states that outer space is not subject to any national appropriation. The ITU is a regulatory body, so it is probably not the appropriate forum to handle the equatorial countries' claim, which rests on jurisdiction questions and on whether the GSO forms a part of the definition of outer space.

The outcome of WARC-ORB 88 exemplifies some of the problems developing countries face in the development of an international regulatory regime for satellite communications sympathetic to their needs and interests. Firstly, the unequal expertise of the negotiating parties can result in an acceptance of administrative procedures and technical standards that reflect the industrialized countries' interests. For example, in the preparation stages of the allotment plan, the developing countries did not have the technical resources to participate in the formulation of the advanced computer models used for the plan. ${ }^{16}$ Typically, in meetings such as the WARCs, officials from developing countries face an array of

14. The BSS already received frequency allotment in 1977 and WARC-ORB 88 added only a feeder link plan to it, so strictly speaking the BSS is not a part of the allotment plan of WARCORB 88.

15. R. JAKHU, in Proceedings of the 83rd Annual Meeting of the American Society of International Law, Chicago (5-8 April 1989), p. 390. 
technical experts from industrialized countries who will explain in great detail why the requirements of the developing countries are not feasible. They will then offer counter-proposals whose implications the developing countries' officials cannot accurately assess on the spot. At WARC-ORB 85 , for instance, experts from the industrialized countries had computer systems on hand, giving immediate detailed assessments of the implications of any proposal that was brought up, while the developing countries were still discussing the need to co-ordinate their positions.

Divisions and differences among the developing countries also prevent them from effectively influencing the negotiations. Countries such as Brazil, China, India, Indonesia and Mexico, initial leaders in the GSO debates, now have implemented their own national satellite networks and consequently do not have the same interests as developing countries without satellite systems. Developing countries with satellite systems tended to be a moderating force of developing country demands to make changes in the ITU regulatory regime because they now have an interest in preserving the protection afforded to their systems under the ITU regime. ${ }^{17}$ These changes may have left the Third World with no clear leadership for post-WARC-ORB 88 discussions.

\section{CONCLUSION}

Since the launching of the first communication satellite in the early 1960s the benefits of space technology have become an essential element in economic, political and social development. For those countries, mostly in the Third World, that have not implemented their own satellite systems, the possibility of preclusion from launching a satellite system and from realizing the benefits of the technology would represent a major handicap in their future national development.

WARC-ORB 88, despite some shortcomings, did create a precedent for the international community to accept some more equal sharing of the limited natural resources of the GSO and frequency spectrum. WARC-ORB 88 illustrated that both developed countries and the Third World can make compromises in an international forum. For the Third World, the WARC-ORB 88 Final Acts represented an important, though limited, step in the international regulation of space communications, although further steps will be needed to fully meet their concerns.

16. Supra n. 13, p. 29. To assemble the plan, the Conference utilized requirements submitted by administrations along with software developed by Japan and the United States. The United States software NASAEC was developed to implement a key aspect of the allotment plan.

17. Supra n. 13, p. 6. 